\title{
Dynamics of polymers in a particle-based mesoscopic solvent
}

\author{
K. Mussawisade, M. Ripoll, R. G. Winkler, a) and G. Gompper \\ Institut für Festkörperforschung, Forschungszentrum Jülich, 52425 Jülich, Germany
}

(Received 8 April 2005; accepted 29 July 2005; published online 10 October 2005)

\begin{abstract}
We study the dynamics of flexible polymer chains in solution by combining multiparticle-collision dynamics (MPCD), a mesoscale simulation method, and molecular-dynamics simulations. Polymers with and without excluded-volume interactions are considered. With an appropriate choice of the collision time step for the MPCD solvent, hydrodynamic interactions build up properly. For the center-of-mass diffusion coefficient, scaling with respect to polymer length is found to hold already for rather short chains. The center-of-mass velocity autocorrelation function displays a long-time tail which decays algebraically as $(D t)^{-3 / 2}$ as a function of time $t$, where $D$ is the diffusion coefficient. The analysis of the intramolecular dynamics in terms of Rouse modes yields excellent agreement between simulation data and results of the Zimm model for the mode-number dependence of the mode-amplitude correlation functions. (0) 2005 American Institute of Physics.
\end{abstract}

[DOI: $10.1063 / 1.2041527]$

\section{INTRODUCTION}

The dynamical behavior of macromolecules in solution is strongly affected or even dominated by hydrodynamic interactions. ${ }^{1,2}$ These interactions have to be taken into account adequately in computer simulations of dilute and semidilute polymer solutions. Such a system can be studied straightforwardly by molecular-dynamics (MD) simulations, in which the fluid particles are modeled in a similar way as the monomers. Due to the large length- and time-scale gap between polymer and solvent dynamics, however, in such an approach almost all CPU time is spent on computing the dynamics of the fluid particles which is in general of minor interest. Nevertheless, such simulations have been performed and provide valuable insight into polymer dynamics. ${ }^{3-6}$

An efficient treatment of the dynamics of complex fluids by computer simulations calls for a coarse grained and simplified description of the solvent dynamics. This has stimulated the development of a number of mesoscale simulation techniques. Prominent examples are lattice gas automata ${ }^{7}$ and lattice-Boltzmann methods, ${ }^{8-12}$ and particle based offlattice methods such as dissipative particle dynamics ${ }^{13-16}$ and multiparticle-collision dynamics ${ }^{17-19}$ (MPCD) (also called stochastic rotation dynamics ${ }^{20}$ ).

The MPCD method has attracted a considerable attention over the last few years and has been applied to a number of colloidal ${ }^{18,21,22}$ and polymeric systems ${ }^{23-28}$ as well as to membranes. ${ }^{29}$ However, no detailed analysis has been performed to which extent MPCD accounts for the hydrodynamic interactions in dilute polymer solutions.

In this article, we study the dynamics of polymer chains in dilute solution with and without intramolecular excludedvolume interactions, where the solvent is modeled by MPCD. To compare simulation results with theories-in particular, the Zimm approach ${ }^{30,31}$ —and scaling predictions, we study the dynamics of Gaussian as well as self-avoiding

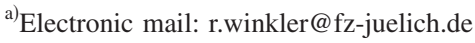

polymers. Our primary goal is to test the ability of a MPCD-MD hybrid method to produce the correct hydrodynamic behavior of polymers in solution.

The transport properties of a MPCD fluid can be tuned by the mean free path of the solvent particles. ${ }^{32}$ Viscous transport dominates over diffusive transport for sufficiently small mean free paths, as is indicated by a large solvent Schmidt number. ${ }^{28,32,33}$ Similarly, the dynamical properties of the polymer depend on the mean free path. Our simulations show that the intramolecular dynamics is much more susceptible to changes in the mean free path than the centerof-mass motion. The reason is that in the first case smaller length-scale properties are probed than by the latter quantity, where the whole molecule contributes. For sufficiently small mean free paths, however, the scaling behavior of the diffusion coefficient and of the correlation functions of the Rousemode amplitudes are obtained in accordance with the predictions of the Zimm model.

Currently attempts are underway to map the detailed atomistic structure of a particular polymer onto a coarse-grained bead-spring model. ${ }^{34-37}$ Typically various chemical groups are mapped onto an effective monomer. Thus, such coarse graining leads to large-size structures with a smaller number of effective monomers. The study of the dynamics of such polymers in dilute solution demands the adequate build up of hydrodynamic interactions even for effectively short chains. Moreover, in simulations of semidilute polymer solutions a sufficiently large number of polymers is required which limits the accessible length of individual polymers. We demonstrate that the MPCD method provides hydrodynamic behavior for such systems.

The paper is organized as follows. In Sec. II the simulation method is outlined and the polymer models are described. The transport properties of the MPCD fluid are described in Sec. III. Results for the simulation of polymers without and with excluded-volume interactions are presented in Sec. IV. Section V summarizes our results. 


\section{SIMULATION METHOD AND MODEL}

The solvent is described as a system of $N$ point particles of equal masses $m$ which are located at the positions $\boldsymbol{r}_{i}(t)$ and possess the velocities $\boldsymbol{v}_{i}(t)(i=1, \ldots, N)$. To maintain a constant particle density, three-dimensional periodic boundary conditions are applied. The MPCD algorithm consists of two steps. In the streaming step, the particles move ballistically. Hence, their positions change according to

$$
\boldsymbol{r}_{i}(t+h)=\boldsymbol{r}_{i}(t)+h \boldsymbol{v}_{i}(t)
$$

in the time interval $h$. We will denote $h$ as the collision time in the following. In the collision step, the particles are sorted into cubic cells of side length $a$. Particles within a collision cell interact with each other through a rotation of the velocity of every particle relative to the center-of-mass velocity $\boldsymbol{v}_{\mathrm{c} . \mathrm{m}}$. of all the particles within that cell. Hence, the velocity of particle $i$ changes according to

$$
\boldsymbol{v}_{i}(t+h)=\boldsymbol{v}_{\mathrm{c} . \mathrm{m} .}(t)+\mathcal{R}(\alpha)\left(\boldsymbol{v}_{i}(t)-\boldsymbol{v}_{\mathrm{c} . \mathrm{m} .}(t)\right),
$$

where $\mathcal{R}(\alpha)$ is the rotation matrix for the rotation by a fixed angle $\alpha$ and $\boldsymbol{v}_{\text {c.m. }}=\sum_{j}^{N_{i} m_{j}} \boldsymbol{v}_{j} / \sum_{j}^{N_{i}} m_{j}$ is the center-of-mass velocity with $N_{i}$ the number of particles in the collision box of particle $i$. The orientation of the rotation axis is chosen randomly for every collision cell and time step. Alternative schemes are also possible. ${ }^{38,39}$ To insure Galilean invariance of the simulation scheme, we apply a random shift for each collision step. ${ }^{20}$ The dynamics of the MPCD algorithm conserves mass, energy, and momentum for each collision cell. There is a $H$ theorem for the algorithm and it yields the correct hydrodynamic equations (Navier-Stokes equation) in the continuum limit. ${ }^{38,40}$

A polymer chain is introduced into the system by adding $N_{m}$ point particles each of mass $M$ which are connected linearly by bonds. We consider two different polymer models, a Gaussian chain and a chain with excluded-volume (EV) interactions. Correspondingly, the following potentials are applied:

(i) Gaussian chain: The monomers are connected by the harmonic potential,

$U_{G}=\frac{3 k_{B} T}{2 l^{2}} \sum_{i=1}^{N_{m}-1}\left(\boldsymbol{r}_{i+1}-\boldsymbol{r}_{i}\right)^{2}$

with zero mean bond length. Here, $l$ is the root-meansquare bond length, $T$ is the temperature, and $k_{B}$ is the Boltzmann constant.

(ii) Excluded-volume chain: The monomers are connected by the harmonic potential,

$U_{\mathrm{EV}}=\frac{\kappa}{2} \sum_{i=1}^{N_{m}-1}\left(\left|\boldsymbol{r}_{i+1}-\boldsymbol{r}_{i}\right|-l\right)^{2}$,

with mean bond length $l$. The force constant $\kappa$ is chosen such that the fluctuations of the bond lengths are on the order of a percent of the mean bond length only. In addition, the monomers interact via the repulsive, truncated Lennard-Jones potential,

$$
U_{\mathrm{RLJ}}=\left\{\begin{array}{l}
4 \epsilon\left[\left(\frac{\sigma}{r}\right)^{12}-\left(\frac{\sigma}{r}\right)^{6}\right]+\epsilon, \quad r>\sqrt[6]{2} \sigma \\
0, \quad \text { otherwise. }
\end{array}\right.
$$

The dynamics of the chain monomers is determined by Newtons' equations of motion between the collisions with the solvent. These equations are integrated by the velocity Verlet algorithm with the time step $h_{p}$. The latter is typically smaller than the collision time $h$. The monomer-solvent interaction is taken into account similar to the solvent-solvent interaction, i.e., the monomers are included in the collision step (2) as suggested in Ref. 23.

We scale length and time according to $\hat{x}=x / a$ and $\hat{t}$ $=t \sqrt{k_{B} T / m a^{2}}$, which corresponds to the choice $k_{B} T=1, m$ $=1$, and $a=1$. The mean free path of a fluid particle $h \sqrt{k_{B} T / m}$ is the given by $\lambda=\hat{h}$. In addition, we set $l=a, \sigma=a$, and $\epsilon / k_{B} T=1$.

\section{TRANSPORT COEFFICIENTS}

In a number of studies, the transport properties of the MPCD fluid have been investigated. ${ }^{17,18,20,32,33,38,40-43}$ In particular, analytical expressions have been derived for the viscosity $\eta$. The total viscosity is the sum of two contributions, the kinetic viscosity $\eta_{\text {kin }}$ and the collisional viscosity $\eta_{\text {coll }}\left(\eta=\eta_{\text {kin }}+\eta_{\text {coll }}\right)$, which explicitly read in three dimensions, ${ }^{38,42}$

$$
\begin{aligned}
& \eta_{\text {coll }}=\frac{m \rho}{18 h a}\left(1-\frac{1}{\rho}\right)(1-\cos \alpha), \\
& \eta_{\text {kin }}=\frac{k_{B} T h \rho}{a^{3}}\left(\frac{5 \rho}{(4-2 \cos \alpha-2 \cos 2 \alpha)(\rho-1)}-\frac{1}{2}\right) .
\end{aligned}
$$

$\rho$ is the number of fluid particles per collision cell and is related to the mass density $\varrho$ by $\varrho=m \rho / a^{3}$. In the derivation of the kinetic contribution, the molecular-chaos assumption is applied. Various numerical studies show that the viscosity agrees very well with the analytical predictions, ${ }^{32,42,43}$ in particular for the range of parameters which we apply in our polymer simulations. It is important to note that the fluid viscosity can be controlled by the average number $\rho$ of fluid particles in a collision cell, since $\eta \sim \rho$ for sufficiently large $\rho$.

Considering a polymer embedded in the solution, we can calculate approximate analytical expressions for the velocity autocorrelation function, the mean-square displacement, and the diffusion coefficient of its center of mass. Similar expression have been obtained for simple fluids in Refs. 32 and 44. Exploiting the molecular-chaos assumption, i.e., the velocity correlation functions among different monomers and between monomers and fluid particles are zero, and neglecting correlations between monomers due to bonds, leads to the polymer center-of-mass velocity autocorrelation function,

$$
\left\langle\boldsymbol{v}_{\text {c.m. }}(n h) \boldsymbol{v}_{\text {c.m. }}(0)\right\rangle=\frac{3 k_{B} T}{M N_{m}}(1-\gamma)^{n},
$$

where 


$$
\begin{aligned}
& \gamma=\gamma_{\alpha} \sum_{k=1}^{\infty} P(k) \frac{\rho m}{k M+\rho m}, \\
& \gamma_{\alpha}=\frac{2}{3}(1-\cos \alpha),
\end{aligned}
$$

with $k$ the number of monomers in a collision cell. The number of monomers per cell varies from cell to cell and as a function of time. Therefore, density fluctuations have to be taken into account. The probability $P(k)$ of a given monomer to be found in a cell with a total of $k-1$ other monomers is given by the Poisson distribution function $P(k)=e^{\phi} \phi^{k-1} /(k$ $-1)$ !, where $\phi$ is the average number of monomers in such a cell. For $M=\rho m$, the average reads

$$
\gamma=\gamma_{\alpha} \frac{1}{\phi^{2}}\left(e^{-\phi}+\phi-1\right) \text {. }
$$

Hence, we find an exponentially decaying correlation function with the characteristic time $\tau_{0}=-h / \ln (1-\gamma)$. The presence of bonds and hydrodynamic interactions will lead to deviations from this simple decay.

With these approximations, the center-of-mass meansquare displacement is found to be

$$
\begin{aligned}
& \left\langle\left(\boldsymbol{r}_{\mathrm{c} . \mathrm{m} .}(n h)-\boldsymbol{r}_{\mathrm{c} . \mathrm{m} .}(0)\right)^{2}\right\rangle \\
& \quad=\frac{6 k_{B} T h^{2}}{M N_{m}}\left[\left(\frac{1}{\gamma}-\frac{1}{2}\right) n-\frac{(1-\gamma)-(1-\gamma)^{n+1}}{\gamma^{2}}\right] .
\end{aligned}
$$

In the long-time limit, $n \rightarrow \infty$, we obtain the diffusion coefficient,

$$
D_{\text {c.m. }}=\frac{k_{B} T h}{M N_{m}}\left(\frac{1}{\gamma}-\frac{1}{2}\right) .
$$

An estimate of the monomer density $\phi$ is found from the radius of gyration $\left(R_{G}\right)$ of a polymer. With $\phi=N_{m} / V$ and $V$ $=4 \pi R_{G}^{3} / 3$, we find $\phi=3 N_{m} /\left(4 \pi R_{G}^{3}\right)$.

\section{POLYMERS IN DILUTE SOLUTION}

To study the dynamics of linear polymers dissolved in a MPCD fluid, we consider chains of various lengths. Most of the results presented below are for polymers of lengths $N_{m}$ $=20$ and $N_{m}=40$, but in some cases we consider polymer lengths up to $N_{m}=160$. The latter is comparable with the polymer length used in the recent Brownian dynamics simulations including hydrodynamic interactions by the RotnePrager tensor. ${ }^{45}$ As our simulations show, certain quantities, like the center-of-mass mean-square displacement (at a certain, finite system size) can be obtained with a moderate expense of computational time even for rather long polymers. The calculation of the scaling behavior of the Rousemode amplitudes, however, requires a significant effort. Thus, we restrict ourselves to the polymer lengths $N_{m} \leqslant 40$ for most aspects. The average number of fluid particles in a collision cell is set to $\rho=10$. Guided by our results on the dynamics of a pointlike heavy particle in the MPCD solvent, ${ }^{44}$ we choose $M=\rho m$. This value provides a sufficiently strong coupling between monomers and fluid to build up hydrodynamic interactions. ${ }^{32}$ The rotation angle is set to $\alpha=150^{\circ}$ and the mean free paths $\lambda=0.1$ and $\lambda=2.0$ are con- sidered. For $\lambda=0.1$, the collisional viscosity dominates over the kinetic viscosity and the total viscosity is $\eta / \sqrt{k_{B} T / m a^{4}}$ $=10$. Hence, the Schmidt number $S c=\eta /(D \varrho)$ is much larger than unity and the simulations are performed in the 'collective regime, ${ }^{32,44}$ where hydrodynamic interactions are taken into acount. On the other hand, for $\lambda=2$ the kinetic contribution to the total viscosity dominates over the collisional viscosity and the Schmidt number is close to unity. Thus, we expect only a partial buildup of hydrodynamic interactions. The long-range nature of the hydrodynamic interaction requires a particular adjustment of the system size in order to extract scaling relations as a function of polymer length. As has been outlined in Ref. 6 , the ratio of the radius of gyration and the systems size has to be constant. Hence, we increase the system size linearly with the increasing radius of gyration $R_{G}$, where $R_{G}=l \sqrt{\left(N_{m}-1\right)\left(N_{m}+1\right) / 6 N_{m}}$ for Gaussian chains and $R_{G} \sim N_{m}^{\nu}$, with $\nu \approx 0.6$, for chains with excluded-volume interactions. For $N_{m}=20$ and no excluded volume interactions, we chose a cubic simulation box of side length $L$ $=18 a$. For chains of the same length interacting via excluded-volume interactions, we used the side length $L$ $=25 a$. Finally, the time step for the velocity Verlet algorithm was set to $\hat{h}_{p}=10^{-2}$ for Gaussian chains and $\hat{h}_{p}=2 \times 10^{-3}$ for polymers with excluded-volume interactions.

\section{A. Gaussian chains}

Simulations of Gaussian chains, i.e., polymers with the bond potential (3), can be compared with analytical calculations based on the Zimm approach. ${ }^{30,31}$ We like to point out, however, that we do not simulate the Zimm model. The Zimm approach relies on the preaveraging approximation, whereas our simulations take into accout the configurational dependence of the hydrodynamic interactions - and therefore thermal fluctuations. The scaling predictions of the Zimm approach have been confirmed experimentally and by computer simulations. ${ }^{31,46-50}$ Thus, the comparison can serve as a test of the simulation method or, vice versa, the validity of the approximations in the Zimm approach.

The equilibrium properties of a polymer are not affected by hydrodynamic interactions. Consistent with that we find excellent agreement between various measured equilibrium quantities, e.g., the radius of gyration of Gaussian chains.

The dynamics of the Gaussian chain without hydrodynamic interactions is described by the well-known Rouse model. ${ }^{31,51}$ Such a dynamics can be realized in our algorithm by considering the polymer only, without any fluid particles. This is achieved, when in the collision step the (full) velocity vector of every individual monomer is rotated around a random axis. The factor $\gamma$ of the velocity autocorrelation function (7) is then exactly given by $\gamma=\gamma_{\alpha}[\mathrm{cf}$. Eq. (9)], since the individual monomer velocities are independent of each other. Simulations of a polymer without fluid particles yield excellent agreement between the numerically determined velocity autocorrelation function and the mean-square displacement with the theoretical expressions (7) and (11) using $\gamma=\gamma_{\alpha}$. Naturally, the autocorrelation functions of the Rouse-mode amplitudes display the predicted behavior too. An alternative scheme for a Brownian-type solvent is used in Ref. 23, 

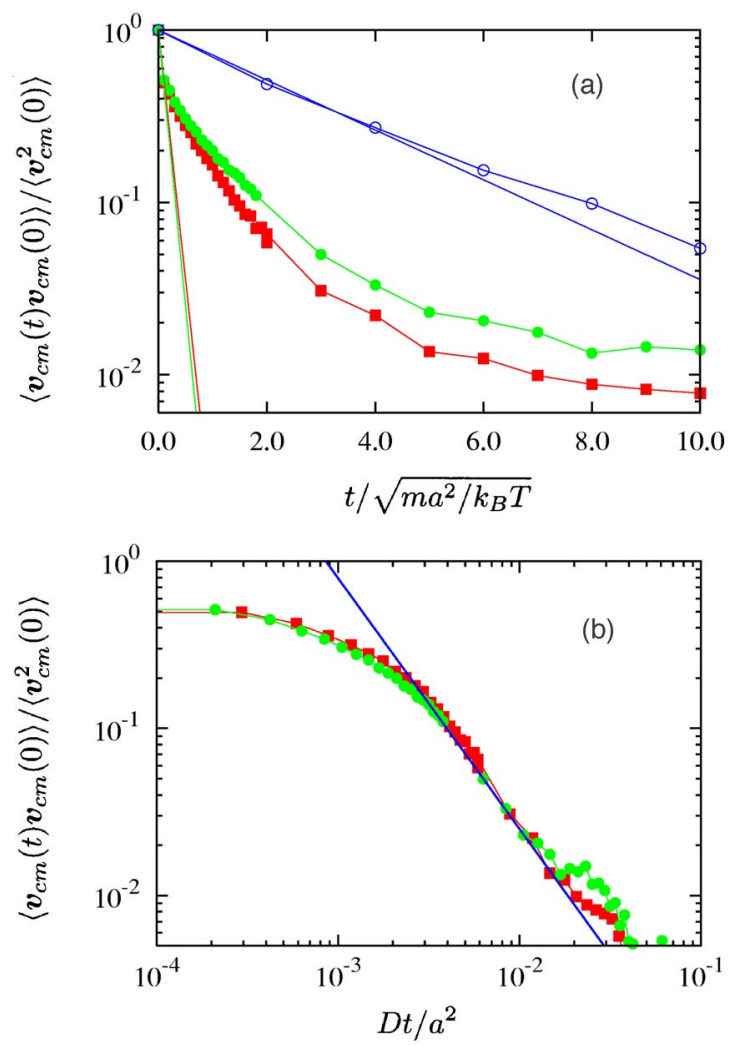

FIG. 1. (Color online.) Center-of-mass velocity autocorrelation functions for Gaussian polymers of length $N_{m}=20$, with the mean free paths $\lambda=0.1$ ( $\square$ ) and $\lambda=2(\bigcirc)$ as well as for $N_{m}=40$ and $\lambda=0.1(\bigcirc)$. (a) The solid lines show the exponential decay predicted by Eq. (7). (b) Log-log representation of the correlation functions for $N_{m}=20, N_{m}=40$, and $\lambda=0.1$ as a function of $D t$. The solid line is proportional to $(D t)^{-3 / 2}$.

where the fluid particles are taken into account and the velocities of the fluid particles are exchanged randomly after every collision step to suppress correlations.

The Zimm model rests upon the Langevin equation for over-damped motion of the monomers, i.e., it applies for times larger than the Brownian time scale $\tau_{B} \gg M / \zeta$, where $\zeta$ is the Stokes friction coefficient. ${ }^{52}$ On such time scales, the velocity correlation functions have decayed to zero and the monomer momenta are in equilibrium with the solvent. Moreover, hydrodynamic interactions between the various parts of the polymer are assumed to propagate instantaneously. This is not the case in our simulations. First of all, the monomer inertia term is taken into account, which implies nonzero velocity autocorrelation functions. Secondly, the hydrodynamic interactions build up gradually. To gain insight into the influence of these aspects on polymer dynamics, Fig. 1(a) displays the center-of-mass velocity autocorrelation function for $N_{m}=20$ and $N_{m}=40$, and for the mean free paths $\lambda=0.1$ and $\lambda=2$. The lines indicate the exponential decay of the correlation function (7) with $\gamma$ of Eq. (10). The monomer density $\phi=3 N_{m}\left(6 / N_{m}\right)^{3 / 2} /\left(4 \pi a^{3}\right)$ has been used, as explained above in Sec. III. The analytical approximation captures the decay of the correlation function for the first collision step (where the molecular-chaos approximation for the solvent is exact) very well, considering the fact that only a rough estimate of the monomer density via the radius of gyration has been used. For the large mean free path $\lambda=2$,

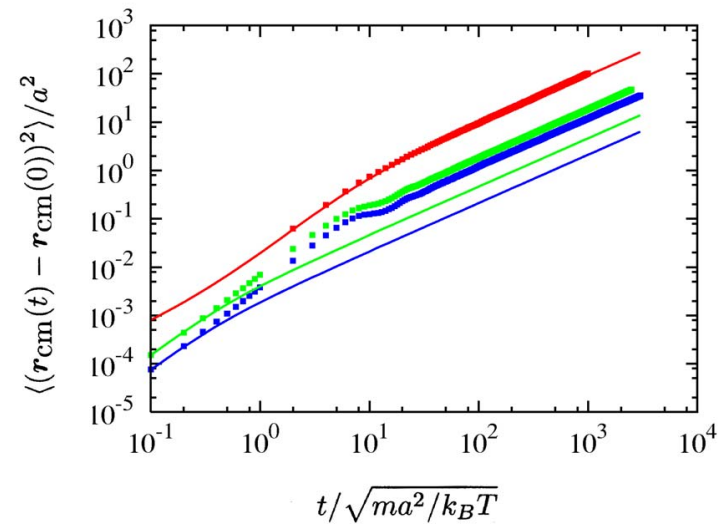

FIG. 2. (Color online.) Center-of-mass mean-square displacements for Gaussian polymers of lengths $N_{m}=20$ and $N_{m}=40$ (symbols). The data are for $N_{m}=20$ and $\lambda=2$ (top), $N_{m}=20$ and $\lambda=0.1$ (middle), and for $N_{m}=40$ and $\lambda=0.1$ (bottom). The lines are calculated according to Eq. (11).

the velocity autocorrelation function is in fact described very well by the exponential decay (7) for about a decade. The nonexponential decay of the correlation functions for $\lambda$ $=0.1$ leads us to the conclusion that in this case hydrodynamic interactions are important in the simulations. Figure 1(b) shows that the correlation function decays as $\left\langle\boldsymbol{v}_{\text {c.m. }}(t) \boldsymbol{v}_{\text {c.m. }}(0)\right\rangle \sim t^{-3 / 2}$ on larger time scales. This algebraic decay has been predicted before and is associated with a coupling between the motion of the polymer and the hydrodynamic modes of the fluid. ${ }^{53-55}$ By scaling the time with the diffusion coefficient $D$, we find that the correlation function is a universal function of $D t$. This is in agreement with the results of dissipative particle dynamics simulations of dilute polymer systems. ${ }^{56}$

The center-of-mass mean-square displacement, which is shown in Fig. 2, exhibits various time regimes. The initial ballistic regime $(t<h)$ is followed by a transient regime, where the inertia effects and the buildup of the hydrodynamic interactions are important. In this time window, the velocity autocorrelation function decays and displays the long-time tail. For longer times, the center-of-mass meansquare displacement increases linearly with time. On such time scales, the velocity autocorrelation function has essentially decayed to zero and even the long-time tail is no longer relevant for the mean-square displacement. Figure 2 displays the center-of-mass mean-square displacements for various polymer lengths and collision times. The lines are calculated using Eq. (11). The hydrodynamic interactions are only weakly developed for $\lambda=2$, since the difference between the simulation data and the analytical approximation-which neglects hydrodynamic interactions-is very small. On the other hand, for the small mean free path $\lambda=0.1$, the hydrodynamic interactions yield a significant increase of the meansquare displacement and hence the diffusion coefficient. As discussed in detail in Refs. 32 and 44, the hydrodynamic interactions are fully developed for small mean free paths only, which is reflected in the deviations from the theoretical expression for the two mean free paths of Fig. 2. In the following, we will always use $\lambda=0.1$, if not otherwise state.

A comparison with the Zimm model can only be under- 
taken in the regime where the mean-square displacement displays a linear time dependence. Figure 2 shows that the linear regime appears for $t / \sqrt{m a^{2} / k_{B} T}>20$ for our fluid parameters. From this linear regime, we obtain the diffusion coefficient for the given size of the simulation box.

The short-time center-of-mass diffusion coefficient in the over damped regime described by the Smoluchowski equation $^{31}$ of a polymer is given by the Kirkwood formula, $1,2,45$

$$
D^{(K)}=\frac{D_{0}}{N_{m}}+\frac{k_{B} T}{6 \pi \eta} \frac{1}{R_{H}},
$$

where the hydrodynamic radius $R_{H}$ is defined as

$$
\frac{1}{R_{H}}=\frac{1}{N_{m}^{2}}\left\langle\sum_{i=1}^{N_{m}} \sum_{j=1}^{N_{m}} \frac{1}{\left|\boldsymbol{r}_{i}-\boldsymbol{r}_{j}\right|}\right\rangle,
$$

and the prime indicates that the term with $j=i$ has to be left out in the summation. For a Gaussian chain, the hydrodynamic radius is found to be $\mathrm{e}^{57}$

$$
R_{H}=\frac{3 l}{8} \sqrt{\frac{\pi}{6}} N_{m}^{1 / 2}\left[1-\frac{3}{4} \zeta(1 / 2) N_{m}^{-1 / 2}+\mathcal{O}\left(N_{m}^{-3 / 2}\right)\right],
$$

where $\zeta(1 / 2)=-1.460 \ldots$. Thus, $R_{H} \sim R_{G}$ to leading order in $N_{m}$, but corrections to scaling are different. In Eq. (13), $D_{0}$ is the diffusion coefficient of a single monomer in the same solvent. Equation (13) applies for a single chain in an infinitely large system only. In a system with periodic boundary conditions, interactions with periodic images appear. We are primarily interested in the scaling behavior of the diffusion coefficient with respect to the hydrodynamic radius and not in its absolute values for a finite system. Therefore, we discuss the diffusion coefficient as a function of the hydrodynamic radius of an infinite system, assuming that $D$ $-D_{0} / N_{m} \sim g\left(R_{G} / L\right) / R_{H}$ as discussed in Ref. 6, where $D$ is extracted from the center-of-mass mean-square displacement.

As is well known, the Kirkwood formula (13) is not equal to $D$ in general. Several effects in a system with fluctuating hydrodynamics lead to a smaller diffusion coefficient. In Refs. 31 and 58 it has been shown that fluctuating hydrodynamic interactions lead to a diffusion coefficient which is a few percent smaller than that with preaveraged hydrodynamic interactions. Moreover, intramolecular dynamic correlations cause a difference between the short- and long-time diffusion coefficients, as has been worked out in detail in Ref. 45. All these considerations are based on the Smoluchowski equation $^{31}$ and the inclusion of hydrodynamic interactions by a tensor. Since our simulations include inertia effects and are not based on a hydrodynamic tensor, additional effects may modify the diffusion coefficient.

The hydrodynamic part $D_{H}=D-D_{0} / N_{m}$ of the diffusion coefficient is presented in Fig. 3 as a function of the hydrodynamic radius (14). In the limit $N_{m} \gg 1$, the diffusion coefficient $D$ is dominated by the hydrodynamic contribution $D_{H}$, since $D_{H} \sim N_{m}^{-1 / 2}$. For shorter chains, $D_{0} / N_{m}$ cannot be neglected, and therefore has to be subtracted in order to extract the scaling behavior of $D_{H}$. The hydrodynamic part of the diffusion coefficient $D_{H}$ exhibits the dependence predicted

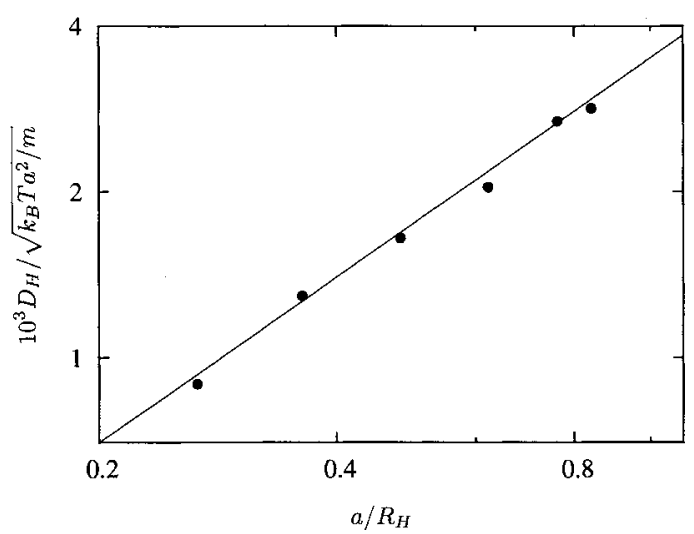

FIG. 3. Dependence of the hydrodynamic part of the diffusion coefficient, $D_{H}=D-D_{0} / N_{m}$, on the hydrodynamic radius for Gaussian chains of lengths $N_{m}=5,10,20,40,80$, and 160 . The mean free path is $\lambda=0.1$.

by the Kirkwood formula and the Zimm theory. Considering finite-system-size corrections to $D$, we find the dependence $D=D_{\infty}-$ const $/ L$ within the accuracy of the simulations, in agreement with the previous studies. ${ }^{2,50,59}$ Increasing the box lengths for polymers of lengths $N_{m}=10,20$, and 40 and extrapolating to an infinite system, we find $D_{0} / \sqrt{k_{B} T a^{2} / m}$ $\approx 1.7 \times 10^{-2}$, which is in agreement with the diffusion coefficient of a monomer in the same solvent. The values $D_{\infty}$ are about $30 \%$ larger than the finite-system-size values presented in Fig. 3.

The Kirkwood formula neglects hydrodynamic fluctuations and is thus identical with the preaveraging result of the Zimm approach. Considering the hydrodynamic part only, the Zimm model yields the diffusion coefficient,

$$
D_{Z}=0.192 \frac{k_{B} T}{\ln \sqrt{N_{m}}} .
$$

For polymers of length $N_{m}=40$ we find $D_{Z} / \sqrt{k_{B} T a^{2} / m}$ $=0.003$. This value agrees with the numerical value for an infinite system: $D_{H} / \sqrt{k_{B} T a^{2} / m}=0.0027$ within $10 \%$. Thus, our simulations yield a diffusion coefficient smaller than $D^{(K)}$ in agreement with previous studies. ${ }^{31,45,58}$ Note that the experimental values are also smaller by about $15 \%$ than those predicted by the Zimm approach. ${ }^{31,60,61}$

The hydrodynamic interactions also affect the intramolecular dynamics. The Zimm approach predicts for the monomer mean-square displacement in the center-of-mass reference frame the dependence, ${ }^{30,31,62}$

$$
\left\langle\left(\left[\boldsymbol{r}_{i}(t)-\boldsymbol{r}_{\mathrm{c} . \mathrm{m} .}(t)\right]-\left[\boldsymbol{r}_{i}(0)-\boldsymbol{r}_{\mathrm{c} . \mathrm{m} .}(0)\right]\right)^{2}\right\rangle \sim t^{2 / 3},
$$

for time scales larger than the transient time determined by the long-time tail and smaller than the Zimm time $\tau_{Z}=\tau_{1}$ $=\eta\left(N_{m} l\right)^{3 / 2} / \sqrt{3 \pi} k_{B} T$, where $\tau_{1}$ is the longest intramolecular relaxation time. This dependence is distinctively different from the behavior of the Rouse model (no hydrodynamic interactions) which predicts a $t^{1 / 2}$ dependence.

Figure 4 displays the monomer mean-square displacement averaged over all monomers. For $\hat{t} \gtrsim 20$, where the mean-square displacement of the center of mass is linear, the monomer dynamics in the center-of-mass reference frame exhibits a behavior close to the predicted power law. Since our polymers are short, we observe a crossover behavior 


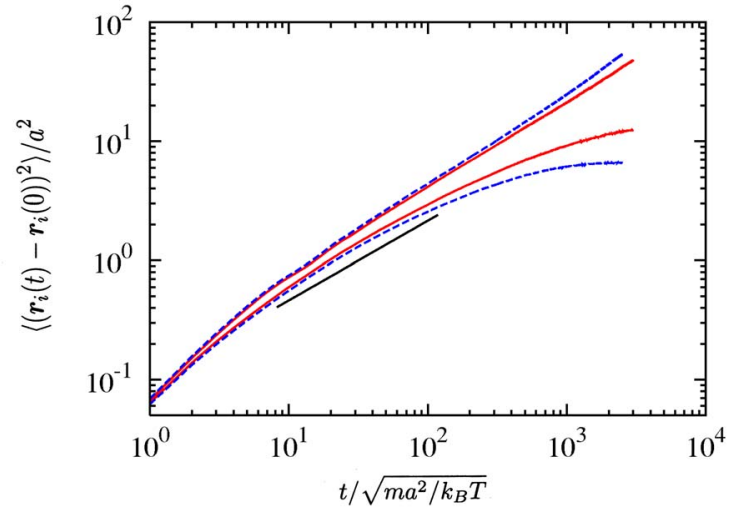

FIG. 4. (Color online.) Monomer mean-square displacements for Gaussian polymers of lengths $N_{m}=20$ (dashed) and $N_{m}=40$ (solid) averaged over all monomers. The top curves correspond to the total mean-square displacement and the bottom ones to the mean-square displacement in the center-of-mass reference frame.

rather than a clear power law. However, the simulation results are consistent with the expected behavior. The total mean-square displacement $\left\langle\left(\boldsymbol{r}_{i}(t)-\boldsymbol{r}_{i}(0)\right)^{2}\right\rangle$ of a monomer displays a somewhat larger slope than that in the center-of-mass reference frame. Only for much longer chains will the total monomer mean-square displacement be close to that in the center-of-mass reference frame, because then the center-ofmass diffusion coefficient will be very small for $t \ll \tau_{Z}$.

To further characterize the internal dynamics of the molecular chain, we perform a mode analysis in terms of the eigenfunctions of the discrete Rouse model. ${ }^{31,51}$ The mode amplitudes are calculated according to

$$
\boldsymbol{\chi}_{p}=\sqrt{\frac{2}{N_{m}}} \sum_{i=1}^{N_{m}} \boldsymbol{r}_{i} \cos \left[\frac{p \pi}{N_{m}}\left(i-\frac{1}{2}\right)\right] .
$$

Due to hydrodynamic interactions, the Rouse modes are no longer eigenfunctions of the chain molecule. However, within the Zimm theory, they are reasonable approximations and the autocorrelation functions of the mode amplitudes decay exponentially, i.e., $\left\langle\boldsymbol{\chi}_{p}(t) \boldsymbol{\chi}_{p}(0)\right\rangle=\left\langle\boldsymbol{\chi}_{p}^{2}\right\rangle \exp \left(-t / \tau_{p}\right)$. For the Rouse model, the relaxation times $\tau_{p}$ depend on chain length and mode number according to $\tau_{p} \sim 1 / \sin ^{2}\left(p \pi / N_{m}\right)$, whereas for the Zimm model the dependence

$$
\tau_{p} \sim\left(p / N_{m}\right)^{1 / 2} / \sin ^{2}\left(p \pi / N_{m}\right)
$$

is obtained. The extra contribution $\sqrt{p / N_{m}}$ follows from the eigenfunction representation of the preaveraged hydrodynamic tensor, under the assumption that its off-diagonal elements do not significantly contribute to the relaxation behavior.

In Fig. 5 the autocorrelation functions for the mode amplitudes are shown for the mean free path $\lambda=0.1$. Within the accuracy of our simulations, the correlation functions decay exponentially and exhibit the scaling behavior according to the Zimm model. Hence, for the small mean free path, hydrodynamic interactions are taken into account correctly. This is no longer the case for the large mean free path $\lambda$ $=2$. Here we observe a scaling behavior in between the predictions of the Rouse and Zimm model. This implies that hydrodynamic interactions are present, but are not fully de-

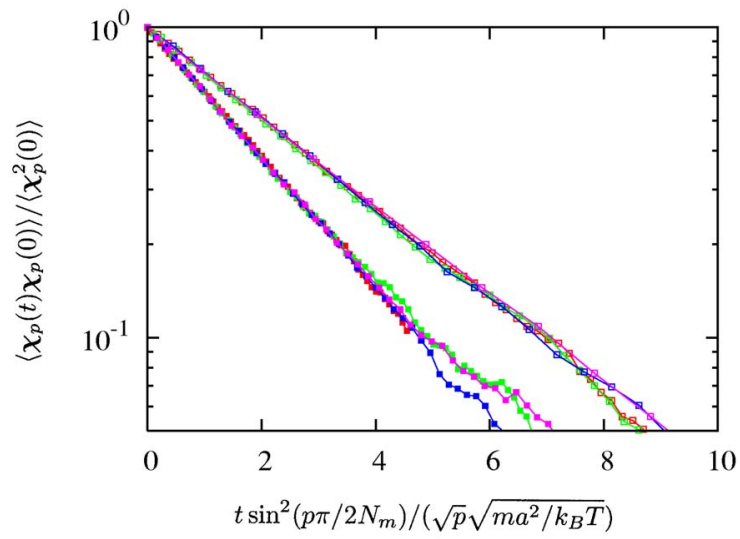

FIG. 5. (Color online.) Correlation functions of the Rouse-mode amplitudes for the modes $p=1-4$ of Gaussian polymers. The chain lengths are $N_{m}$ $=20$ (right) and $N_{m}=40$ (left).

veloped or are small compared to the local friction of the monomers. As mentioned before, we obtain the pure Rouse behavior for a system without solvent by simply rotating the velocities of the individual monomers.

In Ref. 59, a correction term to the standard Zimm result has been derived. Neither is such a correction needed to achieve agreement of our data with the predictions of the Zimm model, nor do our data support the existence of such a term. Hence, we conclude that other approximations in the derivation of the Zimm results compensate for these corrections.

The dependence of the relaxation times on the radius of gyration is displayed in Fig. 6 for $\lambda=0.1$. The scaling behavior $\tau_{1} \sim R_{g}^{3}$ agrees very well with the predictions of the Zimm theory. We even find almost quantitative agreement; the relaxation time of the $p=1$ mode of our simulations is approximately $30 \%$ larger than the Zimm value. ${ }^{31}$

As discussed above, sufficiently long relaxation times compared to the decay of the velocity autocorrelation function are required to observe the dynamics in the Stokes regime. The relaxation times $\tau_{1}$ in Fig. 6 are sufficiently large to fulfill that requirement. Even the relaxation times for higher modes (at least for $p \leqslant 4$ ) are larger than the time required to reach the regime where the mean-square dis-

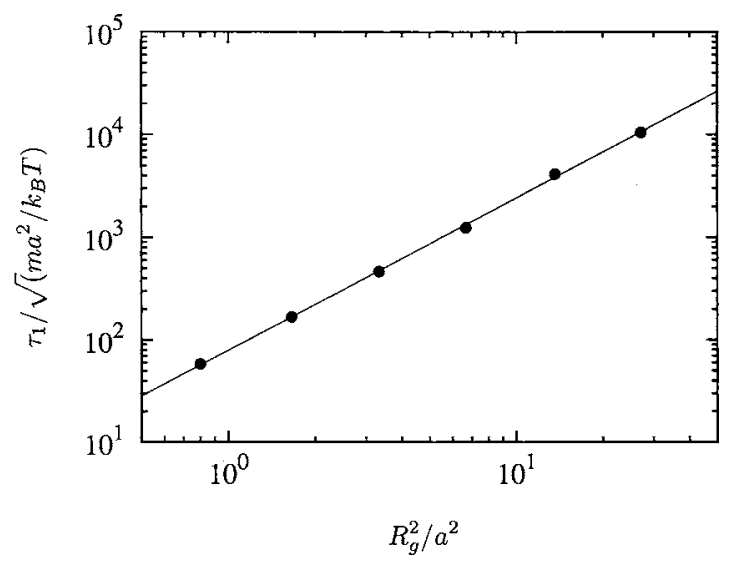

FIG. 6. Dependence of the longest relaxation time $\tau_{1}$ on the radius of gyration for Gaussian chains of the lengths given in Fig. 3. 


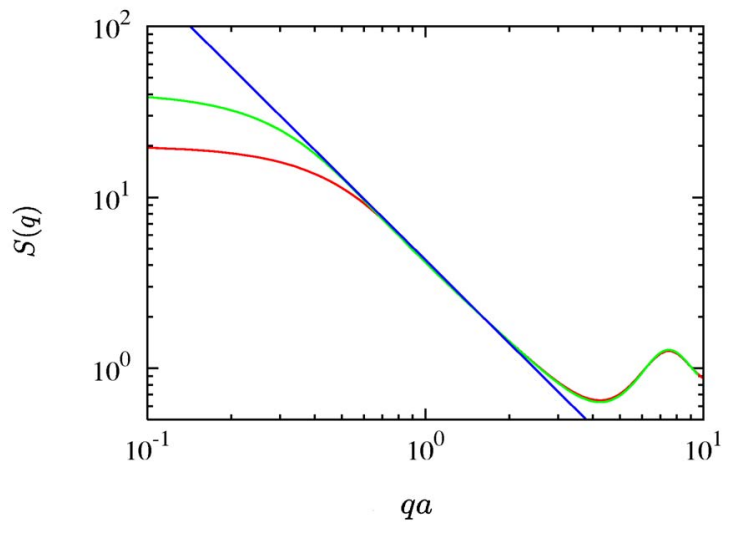

FIG. 7. (Color online.) Single chain static structure factors of polymers with excluded volume interactions of lengths $N_{m}=20$ (bottom) and $N_{m}=40$ (top). The slope of the straight line is approximately -1.6 , which yields a scaling exponent $\nu \approx 0.62$ for the relation $S(\boldsymbol{q}) \sim q^{-1 / \nu}$.

placement of the center of mass of a polymer becomes linear. Note that the relaxation times are proportional to the solvent viscosity and thus can be controlled by the number of fluid particles per collision cell $\rho$.

\section{B. Excluded-volume interactions}

The scaling behavior of equilibrium properties of single polymers with excluded-volume interactions has been studied extensively. ${ }^{31,63-66}$ It has been found that even very short chains $\left(N_{m} \gtrsim 10\right)$ already follow the scaling behavior expected for much longer chains. In particular, the radius of gyration increases like $R_{G} \sim N_{m}^{\nu}$ with the number of monomers, and the static structure factor $S(\mathbf{q})$ exhibits a scaling regime for $2 \pi / R_{G} \ll q \ll 2 \pi / \sigma$, with a $q^{-1 / \nu}$ decay as a function of the scattering vector $q$ and the exponent $\nu \approx 0.6$.

The chain static structure factors for polymers of lengths $N_{m}=20$ and $N_{m}=40$, which are shown in Fig. 7, exhibit a regime which is well described by the decay $q^{-1 / \nu}$ with $\nu$ $\approx 0.62$. The mean-square end-to-end distances and the radii of gyration are consistent with this exponent. Thus, chains of these lengths are reasonably well in the scaling regime for the static properties, in agreement with the previous observations. ${ }^{5,6}$

Considering the dynamics of a polymer chain with excluded-volume interactions, we expect that the diffusion coefficient $D_{H}$ is proportional to $1 / R_{H}{ }^{31}$ similar to the dependence obtained for a Gaussian chain. Thus, the quantity $\left(\left\langle\left(\boldsymbol{r}_{\text {c.m. }}(t)-\boldsymbol{r}_{\text {c.m. }}(0)\right)^{2}\right\rangle-6 D_{0} t / N_{m}\right) R_{H}$ should be independent of the molecular weight of the polymer, at least on large time scales. Figure 8 displays the results for the chain lengths $N_{m}=20$ and $N_{m}=40$. The mean-square displacement is linear at times larger than $\hat{t} \gtrsim 100$ and the two curves fall on top of each other. Hence, the relation for the diffusion coefficient $D_{H} \sim 1 / R_{H}$ also applies for polymers with excluded-volume interactions at the expected time scale.

The mean-square displacement of an individual monomer, averaged over all monomers, is shown in Fig. 9. The simulation data for the two chains lengths are very similar for the total mean-square displacement, but there is essentially no scaling regime $\left(t^{2 / 3}\right)$ for the monomer mean-square

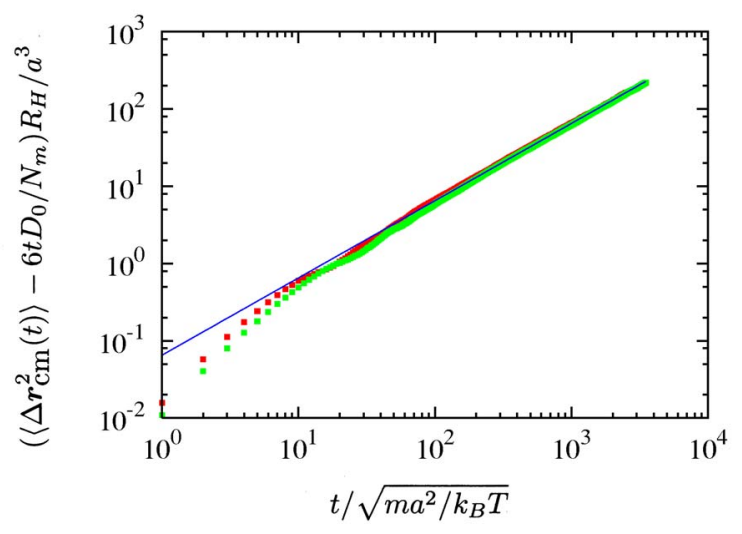

FIG. 8. (Color online.) Center-of-mass mean-square displacements $\left(\boldsymbol{\Delta} \boldsymbol{r}_{\mathrm{c} . \mathrm{m}}\right.$ $\left.=\boldsymbol{r}_{\mathrm{c} . \mathrm{m} .}(t)-\boldsymbol{r}_{\mathrm{c} . \mathrm{m} .}(0)\right)$ as a function of time for polymers with excluded volume interactions of lengths $N_{m}=20$ (top) and $N_{m}=40$ (bottom). The straight line has a slope of 1 .

displacement in the center-of-mass reference frame. This is of course a finite-size effect due to the shortness of the polymers.

To achieve a better theoretical understanding of the simulation results, we map the discrete polymer chain onto a continuous semiflexible polymer model. ${ }^{67}$ We resort to such a description because no analytical result has been presented so far for the dynamics of polymers with excluded-volume interactions. The excluded-volume interactions cause a swelling of the polymer compared to the structure of a phantom chain (freely jointed chain). We account for this increase of the equilibrium chain dimension by the persistence length of the semiflexible chain model. There are various ways to determine the persistence length of the semiflexible chain. One possibility is to consider the correlation of two successive bond vectors $\boldsymbol{R}_{i}$ and to exploit the relation $\left\langle\boldsymbol{R}_{i+1} \boldsymbol{R}_{i}\right\rangle / l^{2}$ $=\exp \left(-l / l_{p}\right)$, where $l_{p}$ is the persistence length. A simple calculation, which takes into account the hard-sphere interactions between nearest and next-nearest neighbors only, yields $\left\langle\boldsymbol{R}_{i+1} \boldsymbol{R}_{i}\right\rangle / l^{2}=\langle\cos \vartheta\rangle$, where $\vartheta$ is the angle between the two adjacent bond vectors, which gives $l_{p}=0.72 l$. However, this

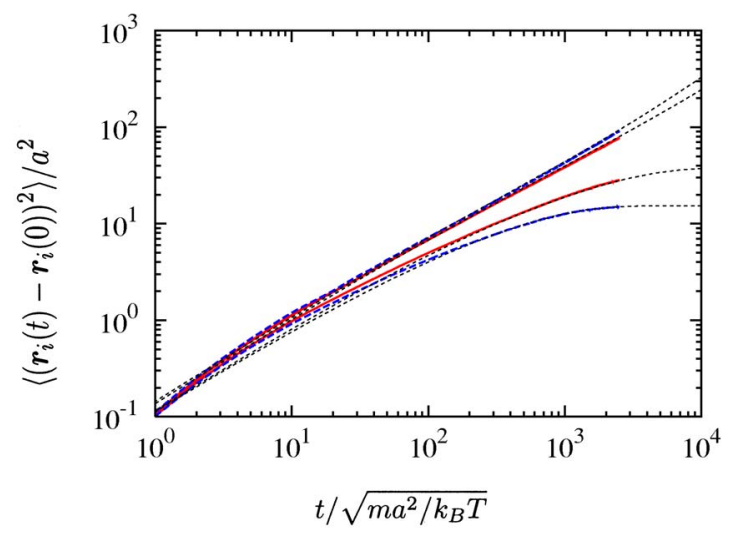

FIG. 9. (Color online.) Monomer mean-square displacements as a function of time for polymers with excluded volume interactions of lengths $N_{m}=20$ and $N_{m}=40$. The top curves represent the mean-square displacements averaged over all monomers, the bottom ones the mean-square displacements in the center-of-mass reference frame. 
value is too small to account for the swelling of the whole molecule, which is due to excluded-volume interactions among the distant monomers along the chain. We mimic this additional excluded-volume effect by adjusting the persistence length such that the radius of gyration of the semiflexible chain agrees with that of the simulated polymer. This procedure yields the ratios $L_{p} / l_{p} \approx 13$, for $N_{m}=20$, and $L_{p} / l_{p} \approx 24$, for $N_{m}=40$. Here, $L_{p}=\left(N_{m}-1\right) l$ is the length of the polymer, and the persistence length corresponds to about 1.6 monomer units. Since the ratios $L_{p} / l_{p}$ are not very large, our simulated polymers should exhibit semiflexible rather than flexible chain behavior. ${ }^{62,68}$ It should be noticed that the choice of a larger persistence length (adjusted to reproduce the radius of gyration) implies that the semiflexible chain is too stiff on the monomer scale; therefore, we cannot expect the model to yield quantitative agreement with the simulation data on short length and time scales.

The dotted lines in Fig. 9 are results of the semiflexible chain model (for details of the model see Refs. 62 and 6870). The theoretical curves describe the simulation data rather well for times $t \gtrsim 100$, i.e., on time scales where the center-of-mass mean-square displacement is linear, which is the time regime where the theoretical model applies. The monomer mean-square displacement in the center-of-mass reference frame does not exhibit any clear scaling regime. The data for the total monomer mean-square displacement seem to show a power-law dependence with time as $t^{0.75-0.78}$. Since no clear power-law regime is seen in the center-ofmass reference frame, we believe that this is a transient behavior rather than a true scaling behavior which reflects the intramolecular dynamics. In Ref. 59, a exponent close to the Zimm value $2 / 3$ has been deduced from simulations of a self-avoiding polymer in a lattice-Boltzmann solvent. Due to the same arguments given above, we believe that they also observe a transient behavior. In conclusion, the considered chains are too short to exhibit the scaling behavior of the monomer mean-square displacement predicted by the Zimm model.

The analysis of the intramolecular dynamics in terms of the Rouse modes yields nonexponentially decaying autocorrelations functions of the mode amplitudes. At short times we find a fast decay which turns into a slower exponential decay. Except for short times, the correlation functions $\left\langle\boldsymbol{\chi}_{p}(t) \boldsymbol{\chi}_{p}(0)\right\rangle /\left\langle\boldsymbol{\chi}_{p}(0)^{2}\right\rangle$ are well fitted by the functions $A_{p} \exp \left(-t / \tau_{p}\right)$, with $A_{1}=0.98, A_{2}=0.973, A_{3}=0.962$, and $A_{4}$ $=0.962$ for $N_{m}=20$ and $A_{1}=0.97, A_{2}=0.97, A_{3}=0.954, A_{4}$ $=0.942$, and $A_{5}=0.93$ for $N_{m}=40$. Figure 10 presents the autocorrelation functions calculated from the simulation data. To emphasize the scaling properties of the correlation functions, they are divided by the respective $A_{p}$. The time axis is scaled with the mode number according to $t p^{\alpha}$, where $\alpha=1.93$ for $N_{m}=20$ and $\alpha=1.85$ for $N_{m}=40$. Within the accuracy of our calculations, the correlation functions exhibit a universal behavior. The Zimm theory predicts the dependence $\tau_{p} \sim p^{-3 v}$ of the relaxation times on the mode number for polymers with excluded-volume interactions. ${ }^{31}$ With $\nu$ $=0.62$, the value following from the analysis of the static quantities, our exponent $\alpha$ for the polymer of length $N_{m}$ $=40$ agrees excellently with the theoretical prediction. The

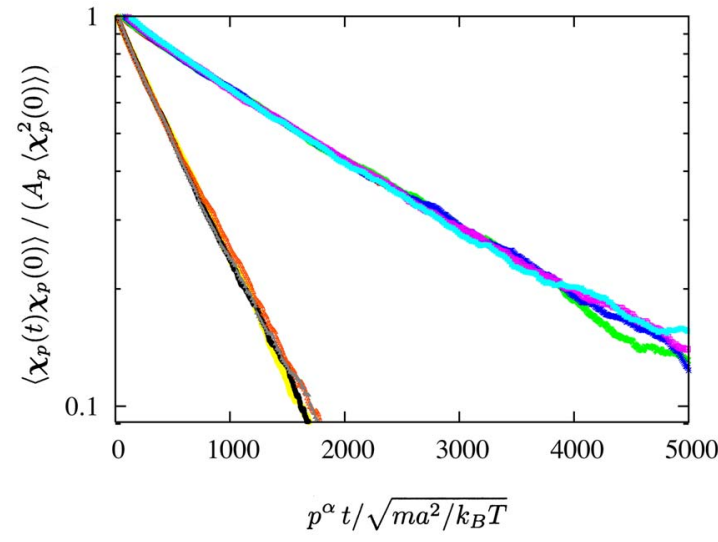

FIG. 10. (Color online.) Correlation functions of the Rouse-mode amplitudes for various modes as a function of the scaled time $t p^{\alpha}$ for polymers with excluded volume interactions. The chain lengths are $N_{m}=20$ (left) and $N_{m}=40$ (right). The calculated correlations where fitted by $A_{p} \exp \left(-t / \tau_{p}\right)$ and have been divided by $A_{p}$. The scaling exponents of the mode numbers are $\alpha=1.93\left(N_{m}=20\right)$ and $\alpha=1.85\left(N_{m}=40\right)$, respectively.

exponent for the polymers with $N_{m}=20$ is slightly larger.

The comparison of the relaxation times extracted from the simulation data with those of the semiflexible chain model for various modes yields excellent agreement (up to $p=5$ ) for both the longer and shorter polymer chains. Calculations exploiting the same polymer model without hydrodynamic interactions yield the exponent $\alpha=2$, corresponding to the Rouse model.

The Zimm model yields the dependence $\tau_{1} \sim N_{m}^{3 \nu}$ of the longest relaxation time on the chain length. ${ }^{31}$ We cannot perform a detailed scaling analysis in this case with our two chain lengths; however, our relaxations times are consistent with this prediction. A direct fit to a single power law gives the dependence $\tau_{1} \sim N_{m}^{1.7}$, i.e., a slightly smaller exponent.

Thus, we find good agreement between the theoretically predicted scaling behavior of the Rouse-mode correlation functions with the simulation results for the modes with long wavelengths. On the other hand, as discussed in the context of the monomer mean-square displacement, we find that the simulated chains are too short to follow the predicted scaling behavior of the intramolecular dynamics. Since the scaling behavior of the monomer mean-square displacement is obtained for $t \ll \tau_{1}$, where $\tau_{1}$ is the longest relaxation time, the agreement of the longest relaxation times with the predictions of the Zimm model does not allow any conclusion on the scaling properties of the monomer mean-square displacement. This implies that the short-wavelength modes, which describe the dynamics on the length scale of a few monomers, are not in the overdamped regime required for the Zimm model to apply.

We finally discuss the scaling behavior of the dynamic structure factor, which is defined as

$$
S(\boldsymbol{q}, t)=\frac{1}{N_{m}} \sum_{i=1}^{N_{m}} \sum_{j=1}^{N_{m}}\left\langle\exp \left(i \boldsymbol{q}\left[\boldsymbol{r}_{i}(t)-\boldsymbol{r}_{j}(0)\right]\right)\right\rangle .
$$

Independent of the solvent conditions $(\Theta$ or good solvent), the Zimm model predicts the scaling relation, ${ }^{31}$ 


$$
S(\boldsymbol{q}, t)=S(\boldsymbol{q}, 0) f\left(q^{\alpha} t\right),
$$

with $\alpha=3$, for $q R_{G} \gg 1$ where the internal motion of the chain is detected. Analytical calculations for Gaussian polymers in the preaveraging approximation yield $\ln f(x) \sim x^{\beta}$ with $\beta=2 / 3$ for $q^{3} t k_{B} T /(6 \pi \eta) \gg 1 .^{31,62,69}$ Thus, $\log (S(\boldsymbol{q}, t) / S(\boldsymbol{q}, 0))$ should follow a straight line in a log-log representation. ${ }^{59}$

The monomer mean-square displacements in the laboratory and in the center-of-mass reference frame are clearly different in the time window $50<t / \sqrt{m a^{2} / k_{B} T}<200$ (cf. Fig. 9), i.e., there is no clear separation of the monomer dynamics and the center-of-mass dynamics. Since the monomer dynamics exhibits a $t^{3 / 4}$ power-law dependence in the laboratory frame, we expect the dynamic structure factor to display a scaling behavior similar to that of a semiflexible polymer, where $S(\boldsymbol{q}, t)=S(\boldsymbol{q}, 0) g\left(q^{8 / 3} t\right)$ for $q R_{G} \gg 1$, rather than that of a flexible polymer. The numerical calculation indeed confirms our assumption and we consistently obtain agreement with this prediction.

To extract the scaling relation for the intramolecular dynamics only, which corresponds to the prediction (21), we resort to the following considerations. As is well known, the dynamic structure factor for a Gaussian distribution of the differences $\boldsymbol{r}_{i}(t)-\boldsymbol{r}_{j}(0)$ and a linear equation of motion is given by ${ }^{31,69}$

$$
\begin{aligned}
S(\boldsymbol{q}, t)= & S(\boldsymbol{q}, 0) \exp \left(-D q^{2} t\right) \\
& \times \frac{1}{N_{m}} \sum_{i=1}^{N_{m}} \sum_{j=1}^{N_{m}} \exp \left(-q^{2}\left\langle\left(\boldsymbol{r}_{i}^{\prime}(t)-\boldsymbol{r}_{j}^{\prime}(0)\right)^{2}\right\rangle / 6\right),
\end{aligned}
$$

where $D q^{2} t$ accounts for the center-of-mass dynamics and $\boldsymbol{r}_{i}^{\prime}$ denotes the monomer position in the center-of-mass reference frame. Strictly speaking, the scaling relation (21) applies for $D q^{2} t \ll 1$, because then the intramolecular dynamics dominates. As mentioned above, for our systems there is no clear separation of the center-of-mass dynamics and the monomer diffusion. To get the dynamics in the center-ofmass reference frame only, we plot $S(\boldsymbol{q}, t) /(S(\boldsymbol{q}, 0)$ $\left.\times \exp \left(-D q^{2} t\right)\right)$, which should follow the scaling prediction (21).

The simulation results for the polymer of length $N_{m}$ $=40$ are shown in Fig. 11. Only $q$ values in the range of $0.7<q a<2.0$ are considered, corresponding to the scaling regime of the static structure factor (cf. Fig. 7) ${ }^{59}$ With respect to the time scale, the interval $50<t / \sqrt{m a^{2} / k_{B} T}<200$ is considered. The numerical results agree very well with the scaling prediction. This is not surprising, since the monomer mean-square displacement in the center-of-mass reference frame exhibits approximately a $t^{2 / 3}$ dependence in the considered time interval (cf. Fig. 9). Thus, our MPCD-MD hybrid simulations are very well suited to study the dynamics of even short polymers in dilute solution.

Ahlrichs and Dünweg reported deviations from the scaling relation (21) in Ref. 59; they achieve the best scaling for the values $\alpha=2.8$ and $\beta=0.71$ in the scaling variable $\left(q^{\alpha} t\right)^{\beta}$. Presumably, the analysis of their results including the diffusion coefficient would yield a scaling of the dynamic structure factor comparable to ours. Considering the other results,

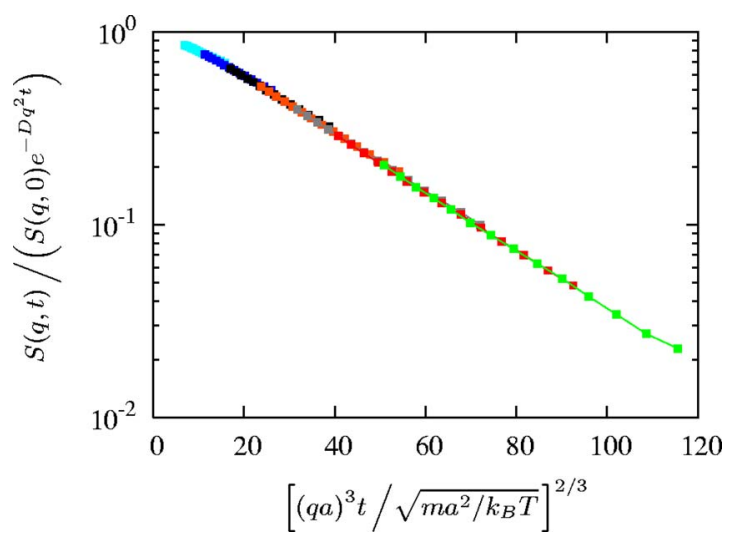

FIG. 11. (Color online.) Normalized dynamic structure factor $S(\boldsymbol{q}, t) /\left(S(\boldsymbol{q}, 0) \exp \left(-D q^{2} t\right)\right)$ of polymers with excluded volume interactions for $N_{m}=40$ and various $q$ values in the range of $0.7<q a<2$ as a function of $q^{2} t^{2 / 3}$.

we conclude that our MPCD hybrid simulations yield comparable results to their lattice Boltzmann-MD hybrid simulations.

Our results for $N_{m}=40$ agree with the results presented by Pierleoni and Ryckaert. ${ }^{5}$ However, we do not observe Zimm scaling for $N_{m}=20$. This is a consequence of the fact that the slope of the monomer mean-square displacement is smaller than $2 / 3$. Naturally, the dynamic structure factor scales, when we use a smaller slope which is consistent with that of the mean-square displacement. In contrast, Pierleoni and Ryckaert obtain consistency with the scaling relation $q^{3} t$ even for short polymers of length $N_{m}=9$ and by using $S(\boldsymbol{q}, t) / S(\boldsymbol{q}, 0)$. This seems to be surprising in the light of our results. However, in Ref. 5 a larger bond length and a higher temperature was used in simulations with explicit solvent. To which extend these differences account for the observations needs further investigation.

Our simulations of polymers confirm the previous conclusions ${ }^{31,59,71}$ that the observation of the scaling behavior predicted by the Zimm model is hampered by short chain lengths. Although the relaxation times for the first few Rouse modes exhibit already the expected scaling behavior, other quantities like the monomer mean-square displacement and the dynamic structure factor display strong finite-size effects. In contrast to the static quantities, e.g., radius of gyration or static structure factor, which exhibit scaling behavior already for rather short polymers, dynamical quantities display scaling relations for much longer polymers only. Using the semiflexible chain model, we predict a Zimm-type regime in the monomer mean-square displacement with respect to the center of mass for polymers which are approximately ten times longer, i.e., for $N_{m} \geq 400$. This is consistent with the observed Zimm-type scaling behavior for polymers with $N_{m}$ $=1000$ in a semidilute solution at short times in Ref. 72 .

\section{SUMMARY AND CONCLUSIONS}

We have presented a study of the dynamics of short polymer chains dissolved in a mesoscopic solvent with and without intramolecular excluded-volume interactions. The solvent dynamics is modeled by multiparticle-collision dynamics. For the interactions between the solvent and a poly- 
mer molecule, a monomer is considered as a point particle and taken into account in the collision step of the MPCD algorithm similar to a fluid particle.

For polymers without excluded-volume interactions, we find excellent agreement of the simulation results with predictions of the Zimm model for various dynamical quantities. In particular, the center-of-mass diffusion coefficient exhibits the dependence $D-D_{0} / N_{m} \sim 1 / R_{H}$ on the hydrodynamic radius. Moreover, the correlation functions of the Rouse-mode amplitudes with long wavelengths decay exponentially and the relaxation times scale according to the predictions of the Zimm model. Similarly, the longest relaxation times display the predicted behavior $\tau_{1} \sim R_{G}^{3}$. Considering the center-ofmass velocity autocorrelation function, we find an algebraic long-time tail which seems to be a universal function of $D t$.

When intramolecular excluded-volume interactions are taken into account, the center-of-mass diffusion coefficient also exhibits the behavior predicted by the Zimm approachor, equivalently, obeys the Kirkwood formula-with respect to the chain length dependence. In addition the correlation functions of the Rouse-mode amplitudes show the modenumber dependence of this model for long wavelength. Considering other quantities, like the monomer mean-square displacement and the dynamic structure factor, we do not find scaling regimes with respect to chain length. The chains with $N_{m}=20$ and $N_{m}=40$ monomers are too short to exhibit the scaling regimes predicted by Zimm theory. The comparison with a semiflexible chain model indicates that considerably longer chains are required to observe the latter behavior. Subtracting the center-of-mass motion, however, we find a consistent scaling behavior of the dynamic structure factor with the monomer mean-square displacement in the centerof-mass reference frame. For $N_{m}=40$, we even obtain agreement with the prediction of scaling theory.

Our simulations show that the MPCD-MD hybrid algorithm for polymers, both with and without excluded-volume interactions, adequately takes into account hydrodynamic interactions in the regime of large Schmidt numbers. In particular, the center-of-mass dynamics can be studied with short chains already and exhibits the universal behavior valid also for long polymers. Treating the monomers as pointlike particles in the interactions with the solvent has no influence on the hydrodynamic interactions. This simplification allows us to efficiently treat the solute-solvent interaction of polymeric systems. The algorithm has the advantage that it can easily be applied to more complex molecules or systems with many polymer chains.

In conclusion, the MPCD algorithm is a powerful method to simulate the dynamics of complex fluids.

\section{ACKNOWLEDGMENTS}

Financial support of this work by the German Research Foundation (DFG) within the SFB TR6 "Physics of Colloidal Dispersion in External Fields" is gratefully acknowledged. One of the authors (M.R.) also acknowledges partial support by the Spanish M.C.Y.T. under the project BFM2001-0290.

${ }^{1}$ J. G. Kirkwood and J. Riseman, J. Chem. Phys. 16, 565 (1948).

${ }^{2}$ J. P. Erpenbeck and J. G. Kirkwood, J. Chem. Phys. 29, 909 (1958).
${ }^{3}$ C. Pierleoni and J.-P. Ryckaert, Phys. Rev. Lett. 61, 2992 (1991).

${ }^{4}$ B. Dünweg and K. Kremer, Phys. Rev. Lett. 66, 2996 (1991).

${ }^{5}$ C. Pierleoni and J.-P. Ryckaert, J. Chem. Phys. 96, 8539 (1992).

${ }^{6}$ B. Dünweg and K. Kremer, J. Chem. Phys. 99, 6983 (1993).

${ }^{7}$ U. Frisch, B. Hasslacher, and Y. Pomeau, Phys. Rev. Lett. 56, 1505 (1986).

${ }^{8}$ G. R. McNamara and G. Zanetti, Phys. Rev. Lett. 61, 2332 (1988).

${ }^{9}$ R. Benzi, S. Succi, and M. Vergassola, Phys. Rep. 222, 145 (1992).

${ }^{10}$ A. J. C. Ladd, J. Fluid Mech. 271, 311 (1994).

${ }^{11}$ S. Chen and G. D. Doolen, Annu. Rev. Fluid Mech. 30, 329 (1998).

${ }^{12}$ P. Ahlrichs and B. Dünweg, Int. J. Mod. Phys. C 9, 1429 (1998).

${ }^{13}$ P. J. Hoogerbrugge and J. M. V. A. Koelman, Europhys. Lett. 19, 155 (1992).

${ }^{14}$ P. Español and P. Warren, Europhys. Lett. 30, 191 (1995).

${ }^{15}$ R. D. Groot and P. B. Warren, J. Chem. Phys. 107, 4423 (1997).

${ }^{16}$ M. Ripoll, M. H. Ernst, and P. Español, J. Chem. Phys. 115, 7271 (2001).

${ }^{17}$ A. Malevanets and R. Kapral, J. Chem. Phys. 110, 8605 (1999).

${ }^{18}$ A. Malevanets and R. Kapral, J. Chem. Phys. 112, 7260 (2000).

${ }^{19}$ A. Lamura, G. Gompper, T. Ihle, and D. M. Kroll, Europhys. Lett. 56, 319 (2001).

${ }^{20}$ T. Ihle and D. M. Kroll, Phys. Rev. E 63, 020201(R) (2001).

${ }^{21}$ S. H. Lee and R. Kapral, Physica A 298, 56 (2001).

${ }^{22}$ E. Falck, J. M. Lahtinen, I. Vattulainen, and T. Ala-Nissila, Eur. Phys. J. E 13, 267 (2004).

${ }^{23}$ A. Malevanets and J. M. Yeomans, Europhys. Lett. 52, 231 (2000).

${ }^{24}$ N. Kikuchi, A. Gent, and J. M. Yeomans, Eur. Phys. J. E 9, 63 (2002).

${ }^{25}$ E. Falck, O. Punkkinen, I. Vattulainen, and T. Ala-Nissila, Phys. Rev. E 68, 050102 (2003).

${ }^{26}$ S. Y. Ko and S. H. Lee, Bull. Korean Chem. Soc. 24, 771 (2003).

${ }^{27}$ I. Ali, D. Marenduzzo, and J. M. Yeomans, J. Chem. Phys. 121, 8635 (2004).

${ }^{28}$ R. G. Winkler, K. Mussawisade, M. Ripoll, and G. Gompper, J. Phys.: Condens. Matter 16, S3941 (2004).

${ }^{29}$ H. Noguchi and G. Gompper, Phys. Rev. Lett. 93, 258102 (2004).

${ }^{30}$ B. H. Zimm, J. Chem. Phys. 24, 269 (1956).

${ }^{31}$ M. Doi and S. F. Edwards, The Theory of Polymer Dynamics (Clarendon, Oxford, 1986)

${ }^{32}$ M. Ripoll, K. Mussawisade, R. G. Winkler, and G. Gompper, Europhys. Lett. 68, 106 (2004).

${ }^{33}$ T. Ihle and D. M. Kroll, Phys. Rev. E 67, 066706 (2003).

${ }^{34}$ M. Müller, J. P. Wittmer, and J.-L. Barrat, Europhys. Lett. 52, 406 (2000).

${ }^{35}$ M. Pütz, K. Kremer, and G. S. Grest, Europhys. Lett. 49, 735 (2000).

${ }^{36}$ J. T. Padding and W. J. Briels, J. Chem. Phys. 117, 925 (2002).

${ }^{37}$ D. Reith, M. Pütz, and F. Müller-Plathe, J. Comput. Chem. 24, 1624 (2003).

${ }^{38}$ E. Tüzel, M. Strauss, T. Ihle, and D. M. Kroll, Phys. Rev. E 68, 036701 (2003).

${ }^{39}$ E. Allahyarov and G. Gompper, Phys. Rev. E 66, 036702 (2002).

${ }^{40}$ C. M. Pooley and J. M. Yeomans, J. Phys. Chem. B 109, 6505 (2005).

${ }^{41}$ T. Ihle and D. M. Kroll, Phys. Rev. E 67, 066705 (2003).

${ }^{42}$ N. Kikuchi, C. M. Pooley, J. F. Ryder, and J. M. Yeomans, J. Chem. Phys. 119, 6388 (2003).

${ }^{43}$ T. Ihle, E. Tüzel, and D. M. Kroll, Phys. Rev. E 70, 035701 (2004).

${ }^{44}$ M. Ripoll, K. Mussawisade, R. G. Winkler, and G. Gompper, Phys. Rev. E 72, 016761 (2005)

${ }^{45}$ B. Liu and B. Dünweg, J. Chem. Phys. 118, 8061 (2003).

${ }^{46}$ T. A. King, A. Knox, W. I. Lee, and J. D. G. McAdam, Polymer 14, 151 (1973).

${ }^{47}$ M. Adam and M. Delsanti, Macromolecules 10, 1229 (1977).

${ }^{48}$ T. Nose and B. Chu, Macromolecules 12, 590 (1979).

${ }^{49}$ C. C. Han and A. Z. Akcasu, Macromolecules 14, 1080 (1981).

${ }^{50}$ N. A. Spenley, Europhys. Lett. 49, 534 (2000).

${ }^{51}$ P. E. Rouse, J. Chem. Phys. 21, 1272 (1953).

${ }^{52}$ J. K. G. Dhont, An Introduction to Dynamics of Colloids (Elsevier, Amsterdam, 1996).

${ }^{53}$ M. H. Ernst, E. H. Hauge, and J. M. J. van Leeuwen, Phys. Rev. Lett. 25, $1254(1970)$

${ }^{54}$ M. H. Ernst, E. H. Hauge, and J. M. J. van Leeuwen, Phys. Rev. A 4, 2055 (1971)

${ }^{55}$ J.-P. Hansen and I. R. McDonald, Theory of Simple Liquids (Academic, London, 1986).

${ }^{56}$ C. P. Lowe, A. F. Bakker, and M. W. Dreischor, Europhys. Lett. 67, 397 (2004). 
${ }^{57}$ B. Dünweg, D. Reith, M. Steinhauser, and K. Kremer, J. Chem. Phys. 117, 914 (2002).

${ }^{58}$ M. Fixmann, J. Chem. Phys. 78, 1594 (1983).

${ }^{59}$ P. Ahlrichs and B. Dünweg, J. Chem. Phys. 111, 8225 (1999).

${ }^{60}$ M. Schmidt and W. Burchard, Macromolecules 14, 210 (1981).

${ }^{61}$ W. H. Stockmayer and B. Hammouda, Pure Appl. Chem. 56, 1373 (1984).

${ }^{62}$ L. Harnau, R. G. Winkler, and P. Reineker, J. Chem. Phys. 104, 6355 (1996).

${ }^{63}$ P.-G. de Gennes, Scaling Concepts in Polymer Physics (Cornell University, Ithaca, 1979).

${ }^{64}$ J. des Cloizeaux and G. Jannink, Polymer Solutions: Their Modelling and Structure (Clarendon, Oxford, 1990).
${ }^{65}$ D. Ceperly, M. H. Kalos, and J. L. Lebowitz, Macromolecules 14, 1472 (1981).

${ }^{66}$ K. Kremer and K. Binder, Comput. Phys. Rep. 7, 261 (1988).

${ }^{67}$ R. G. Winkler, P. Reineker, and L. Harnau, J. Chem. Phys. 101, 8119 (1994).

${ }^{68}$ L. Harnau, R. G. Winkler, and P. Reineker, Europhys. Lett. 45, 488 (1999).

${ }^{69}$ R. G. Winkler, L. Harnau, and P. Reineker, Macromol. Theory Simul. 6, 1007 (1997).

${ }^{70}$ R. G. Winkler, S. Keller, and J. O. Rädler (unpublished).

${ }^{71}$ J. des Cloizeaux, J. Phys. (Paris), Lett. 39, L151 (1978).

${ }^{72}$ P. Ahlrichs, R. Everaers, and B. Dünweg, Phys. Rev. E 64, 040501 (2001). 